\section{Ein Leben retten. 100 Pro Reanimation}

eln Leвen 16.9.-22.9.2013 reTten WOCHE DER 100 Pro WIEDERBELEBUNG

www.einlebenretten.de

\author{
Hugo Van Aken
}

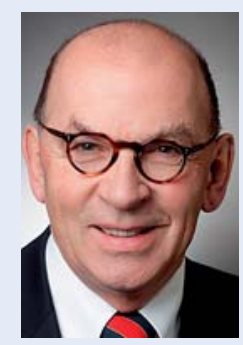

Hugo Van Aken
B

ereits 3-5 Minuten nach einem Herzkreislaufstillstand treten unwiderrufliche Schäden des Gehirns auf. Minuten, welche über ein Menschenleben entscheiden können. Obwohl der Herzstillstand in ca. $60 \%$ der Fälle beobachtet wird, beginnen in Deutschland weniger als $18 \%$ der Umstehenden mit Wiederbelebungsmaßnahmen und übernehmen damit mechanisch die Funktion des Herzens, um den Körper und damit das Gehirn mit Sauerstoff zu versorgen. Da der professionelle Rettungsdienst zwar schnell, jedoch nahezu nie innerhalb von 5 Minuten beim Patienten sein kann, ist man in genau diesen entscheidenden Minuten auf die Maßnahmen der Ersthelfer angewiesen. In einer Studie von Böttiger und Mitarbeiter konnte festgestellt werden, dass die Überlebensrate nach kardial bedingtem Kreislaufstillstand nach 1 Jahr ohne Laienreanimation $10 \%$ betrug und, wenn die Laienreanimation bereits begonnen wurde, bevor der professionelle Rettungsdienst eingetroffen war, konnte die Überlebensrate auf 18\% gesteigert werden [1].

Somit ist es zur Steigerung der Überlebensrate enorm wichtig, die Aufmerksamkeit der Bevölkerung für den lebensbedrohlichen „Herzstillstand“ zu erhöhen und die Laien in Wiederbelebungsmaßnahmen der sog. Reanimation zu schulen. Den Daten des Deutschen Reanimationsregisters ist zu entnehmen, dass es in Deutschland ca. 70.000 erfolglose Wiederbelebungsmaßnahmen pro Jahr gibt. Durch Laienreanimation steigt die Überlebensrate von $10 \%$ auf $18 \%$. Dies bedeutet, dass $\mathbf{5 . 6 0 0}$ Menschenleben jährlich gerettet werden könnten. Zum Vergleich: Die Zahl der Verkehrstoten pro Jahr liegt nach Angabe des statistischen Bundesamtes bei ca. 3.200.

Deutschland hat hier einen hohen Nachholbedarf: In den meisten Ländern der EU liegt diese Rate deutlich höher. In Schweden und Norwegen beispielsweise machen $60 \%$ der Bevölkerung im Notfall eine Herzdruckmassage. Dabei geht es meist keineswegs darum, schwerverletzte Fremde zu retten. Etwa 70\% der Herzstillstände treten in häuslicher Umgebung auf und sind nicht durch Verletzungen bedingt. „Was sich liebt, das drückt sich!“

Jeder kann zum „Augenzeugen“ und also auch zum potenziellen Lebensretter werden. Eben genauso, wie der plötzliche Herztod bzw. Herzstillstand jeden treffen kann. Vornehmlich sind zwar diejenigen mit Risikofaktoren, wie Übergewicht, Bluthochdruck, Verengung der Herzkranzgefäße oder Herzinfarkt, gefährdet, aber auch jüngere Menschen sind nicht vor dem plötzlichen Herztod sicher.

Die aktuellen Leitlinien zur Cardio-Pulmonalen-Reanimation des European Resuscitation Councils bestätigen die hohe Bedeutung der Basismaßnahmen Herzdruckmassage und Beatmung für das gute neurologische Überleben von Patienten mit einem Herzkreislaufstillstand. Die unterbrechungsfreie und qualitativ hochwertige Durchführung der Herzdruckmassage steht dabei im Vordergrund. Basismaßnahmen durch den anwesenden Laien ohne besondere Hilfsmittel liefern hier einen großen Beitrag zum Überleben nach einem präklinischen Herzkreislaufstillstand.

Mit der Kampagne „100 Pro Reanimation“, die die Deutsche Gesellschaft für Anästhesiologie und Intensivmedizin und des Berufsverband Deutscher Anästhesisten im Jahr 2012 gestartet haben, soll verdeutlicht werden, dass jeder mit ganz einfachen Maßnahmen einem Menschen das Leben retten kann. Gemeinsam mit dem German Resuscitation Council und der Stiftung Deutsche Anästhesiologie wollen beide Verbände vom 16. -22. September diesen Jahres das Thema Laienreanimation mit der „Woche der Wiederbelebung" unter der Schirmherrschaft des Bundesministeriums für Gesundheit und einer breit angelegten Medienkampagne in die Öffentlichkeit tragen. Die Ängste, etwas falsch machen zu können, sollen abgebaut werden; Ekelfaktoren vor der Mund-zu-MundBeatmung genommen werden, da diese Maßnahme gegenüber der Herzdruckmassage in den Hintergrund treten kann [2]. 


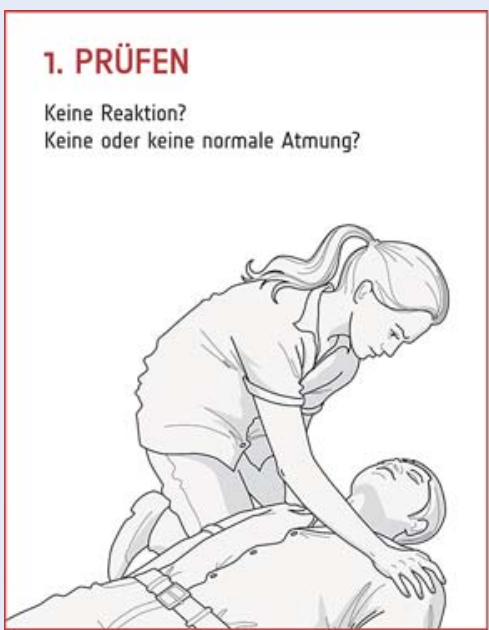

\section{RUFEN}

Rufen Sie 112 an.

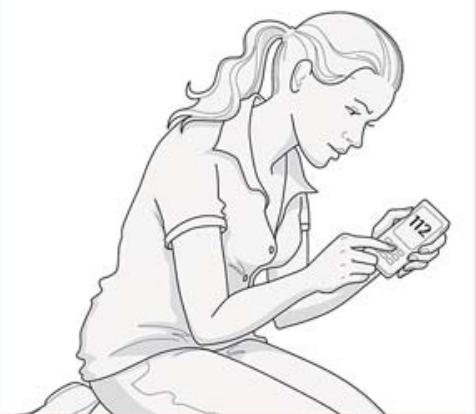

\section{DRÜCKEN}

Drücken Sie fest und schnell in der Mitte des Brustkorbs: mind. 100 x pro Minute. Hören Sie nicht auf, bis Hilfe eintrifft.

Abb. 1 Schaubild Prüfen-Rufen-Drücken

Oft wird aus Angst, etwas falsch zu machen und aus Unwissenheit welche Maßnahmen richtig sind, gar nicht geholfen. Dabei ist der einzige Fehler, der im Fall eines Herzstillstands gemacht werden kann, nichts zu tun. Denn die Maßnahmen sind einfach - und Sie können nichts falsch machen! Durch 3 Maßnahmen können auch Sie einem vom Herzstillstand Betroffenen das Leben retten: Die drei leicht zu merkenden Worte der Wiederbelebung lauten: Prüfen-Rufen-Drücken (Abb. 1).

\section{Literatur}

1 Böttiger BW, Grabner C, Bode C et al. Long term outcome after out-of-hospital cardiac arrest with physican staffed emergency medical services:the Utstein style applied to midsized urban/suburban area. Heart 1999; 82: 674-679

2 Wolcke B. Kontroversen in der Notfallmedizin: Laienreanimation mit und ohne Beatmung. Anästhesiologie, Intensivmedizin, Notfallmedizin, Schmerztherapie 2013; 48: im Druck

Korrespondenzadresse

Prof. Dr. Hugo Van Aken

Klinik für Anästhesiologie, operative Intensivmedizin und Schmerztherapie

Universitätsklinikum Münster

Albert-Schweitzer-Campus 1, Gebäude A1

48149 Münster

hva@uni-muenster.de 